\title{
Torneo de microfutbol: un encuentro deportivo entre los integrantes de la gran familia estomatológica.
}

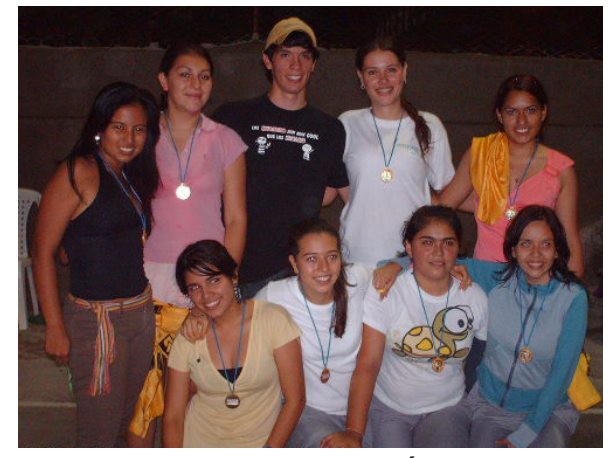

Campeonas: Lorena García, Ángela Zúñiga, Laura Castillo, Alejandra Moreno, Ana María Valencia, Lesly Palacios, Alejandra Ordoñez, Alexandra lagos.

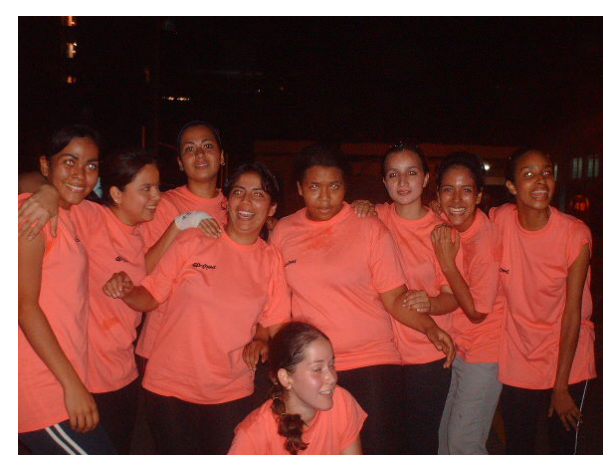

Maryuri Salguero, Anyela Osorio, María Espinosa, Paola Bolaños, Lorena Mora, Ceferina Valencia, Nataly Marmolejo, Lina Carmona, Pilar León.

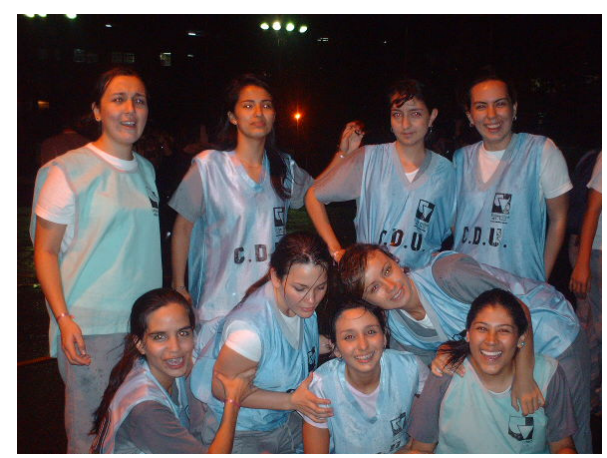

Ángela Zapata, Nazly Narváez, Zugey Zúñiga, Sofía Salas, Ángélica Castillo, Katherine Cardona, Darling Lozada, María Fernanda Mosquera.

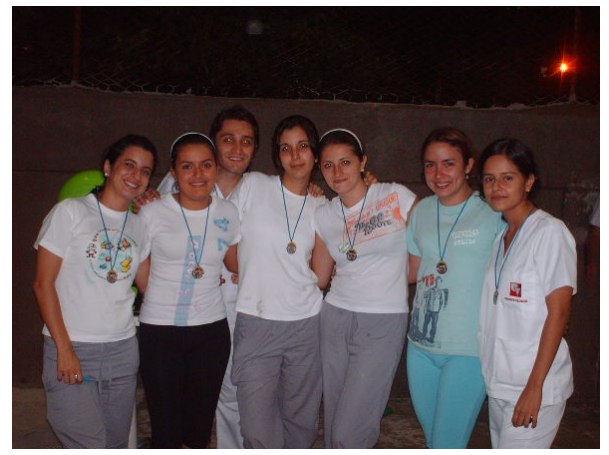

Sub-campeonas: Tatiana Arias, Bibiana Pazos, Ingrid Zamora, Ángela Benavides, Claudia Rincón, Sofía Villareal.

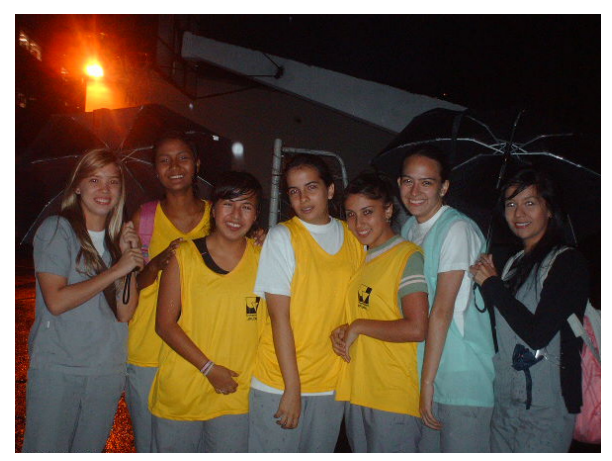

Natalia Correa, Wanda Gómez, Andrea Ochoa, Catalina García, Verónica Parra, Erika Melo, Yesica Pardo, Eliana Mejía, Carolina López.

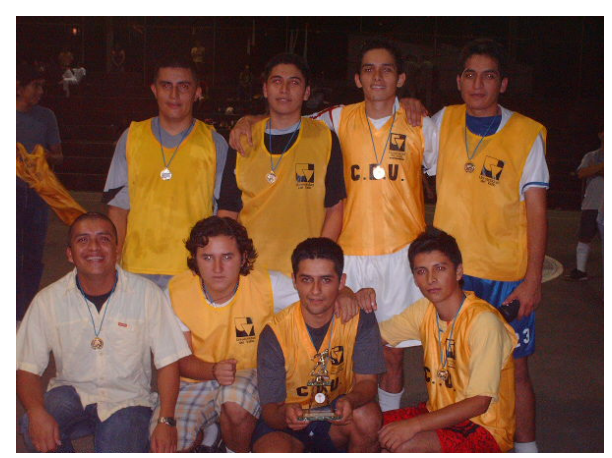

Campeones: Cristian Herrera, Oscar Chicaiza, Jerson López, Kevin Gómez, Camilo Quintero, Dario Cifuentes, Alexander González, Mario Castillo.
La Federación Odontológica Estudiantil de la Universidad del Valle F.O.E.V., organizó dos torneos de microfutbol (uno femenino y otro masculino) en el Centro Deportivo Universitario C.D.U. de la Universidad del Valle Sede San Fernando en donde se congregó, en torno al balón, la gran familia estomatológica integrada por los profesores, personal administrativo, estudiantes de odontología, postgrado e higiene oral y egresados.

Durante varias semanas se jugaron cerca de 30 partidos en donde el compañerismo, el sentido de pertenencia por la Escuela de Odontología y el juego limpio sobresalieron por encima de los resultados adversos, no solo en la cancha, sino tambien en las tribunas, en donde las barras "bravas" de cada equipo apoyaron a los jugadores sin dar paso a insultos o vejaciones.

Los que asistieron a ver los partidos desde la tribuna, disfrutaron de un excelente nivel técnico y táctico en cada partido y los que participaron en los equipos encontraron la disculpa para volver a hacer alguna actividad física (para el caso de los profesores y egresados) y para satisfacer una necesidad vital tanto de hombres y mujeres como lo es patear una pelota de fútbol.

Gracias al profundo sentido de pertenencia de los asistentes, a la entrega de los jugadores en la cancha y a la excelente organización de la junta directiva de la F.O.E.V., se pudo desarrollar dos grandes torneos de que procuraron la integración y sana diversión de los miembros de la gran familia estomatológica.

Ante ello, la Revista Estomatología rinde tributo a todas esas personas, artífices y visionarios del día a día, que participaron de una u otra forma en la organización y puesta en marcha de dichos eventos.

\section{Revista Estomatología}




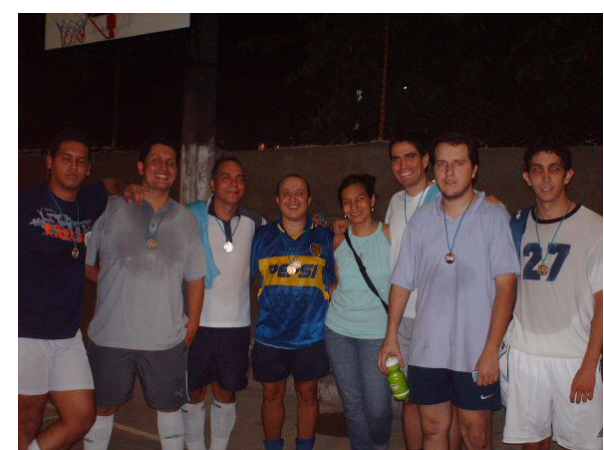

Sub-campeones: Julian Balanta, Jorge Quisoboni, Álvaro Abadía, Pablo Molano, Mauricio Gallego, Freddy Moreno, Óscar Zuluaga, Herney Garzón, Carlos Buriticá.

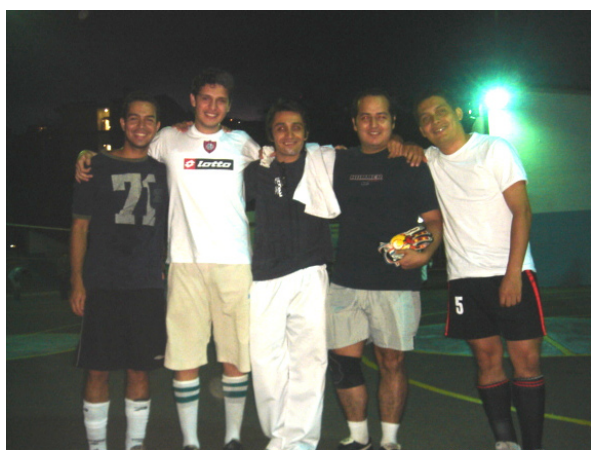

John Angel Rodríguez, Alejandro Rendón, Andrés González, Carlos Piedrahita, Giovany Beltrán, Vladimir Ceballos, Carlos Muñoz, Christian Castillo, Mario Múnera.

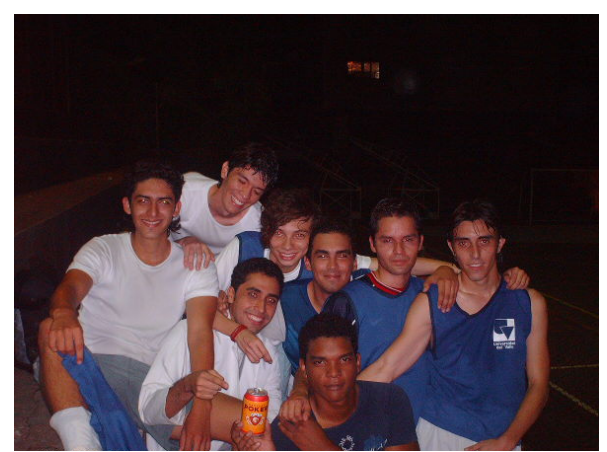

Esteban Puellar, Daniel Gómez, Juan Pablo Pava, Juan Alejandro Arias, Carlos Gutierrez, John Edward marulanda, Adrian Velasco, Andrés Felipe Navarrete.

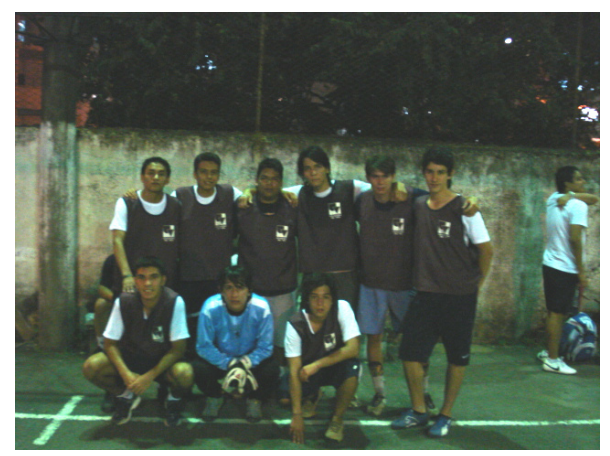

David Piarpuzán, Bruno Gutiérrez, Felipe Plaza, Carlos Bonnelo, Julio César Chavez, Esteban Londoño, Pablo Pitto, Julián Medina.

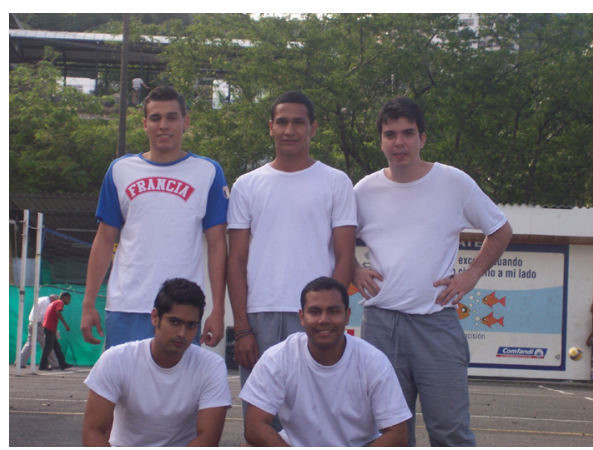

Marlon Belalcazar, Jaime Toro, Jaime García, Pedro Castro, José Manuel Martínez, Alexander Salguero, Juan David Sánchez, Andrés Cruz, Jorge García.

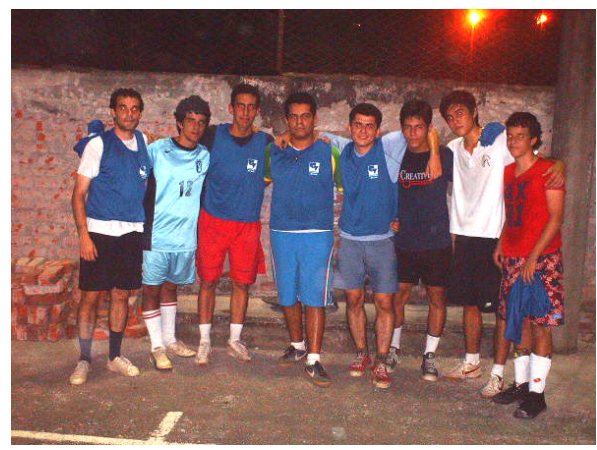

Jonathan Rubio, Rodrigo Ramírez, Mauricio Rubio, Camilo Arteaga, Harold Arboleda, Julián Puerta, Luis Torres, José Díez, Sergio Pérez, Carlos Gallego.

\section{Viviana Pasos}

Presidente Federación Odontológica Estudiantil de la Universidad del Valle F.O.E.V. 2006-2007. 